\title{
The ILOG JViews Graph Layout Module
}

\author{
Georg Sander and Adrian Vasiliu \\ ILOG SA, 9, rue de Verdun - BP 85, 94253 Gentilly Cedex, FRANCE \\ \{sander|vasiliu\}@ilog.fr, http://jviews.ilog.com
}

\section{Short Description}

The ILOG JViews Component Suite is a set of pure Java components for building sophisticated interactive commercial Web-based user interfaces. Besides the graphics, interaction, and animation framework, the graph layout module is one of the key components.

\section{Areas of Application}

Applications of the ILOG JViews Component Suite range from Telecom (network display) over Geographic Information Systems (cartographic maps) to Resource Scheduling (Gantt charts and workflow diagrams). It contains a broad set of layout algorithms for the automatic arrangement of different kinds of diagrams such as PERT charts, process and workflow diagrams, WAN and LAN networks, and supply chain diagrams.

\section{Layout Algorithms and Layout Features}

The ILOG JViews graph layout module includes the following features:

- Topological Mesh Layout: Designed for biconnected graphs as they occur in database and knowledge engineering. It arranges outer nodes on a circle and tries to expose symmetries of inner nodes 4 .

- Spring Embedder Layout: A force-directed algorithm [1] that distributes nodes evenly in a specified area.

- Uniform Length Edges Layout: Another force-directed algorithm that arranges the graph such that all edges have approximately the same length.

- Tree Layout: An incremental algorithm based on [5] to arrange trees. Besides the classical tree style, it includes a tip-over mode (children are arranged sequentially instead of in parallel to optimize the area) and a radial mode (nodes are placed in circles/ellipses around the root).

- Hierarchical Layout: Flow layout based on partitioning into layers, with support for various edge routing styles [3, connection ports and constraints.

- Bus Layout: Designed for bus topologies in networking and telecommunications.

- Circular Layout: It displays ring and star network topologies interconnected in a tree structure for telecommunication applications. 
- Grid Layout: Arranges disconnected nodes in rows, columns, or on a grid. It is also utilized to arrange the connected components of graphs.

- Link Layout: An edge routing algorithm that doesn't move the nodes. It supports orthogonal and direct edge shapes and offers two modes: a fast combinatoric algorithm for short edges and a classical obstacle/maze routing for long edges similar to the PCB and VLSI routing algorithms [2].

- Automatic Label Placement: A fast simulated annealing technique to place labels at nodes and edges while avoiding overlaps.

- Nested Layout: The layout algorithms can be combined in multiple ways to arrange nested graphs (that is, graphs with nodes that contain graphs).

\section{Architecture}

The ILOG JViews Graph Layout framework contains extensive functionality (a generic graph model, 13 layout algorithms, notification mechanisms, graph filters, etc.). The framework is a set of extensible Java classes that can easily be integrated into customer applications. ILOG also provides ready-to-use components built upon the graph layout module, such as the ILOG JViews for Workflow Applications or the ILOG Telecom Graphic Objects for Java.

\section{Screenshots}

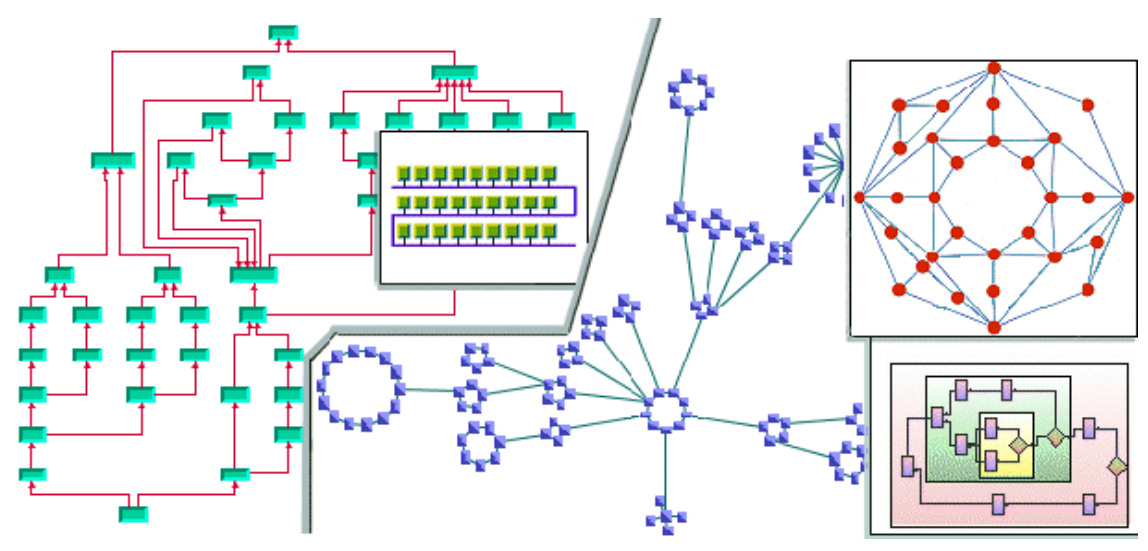

\section{References}

1. P. Eades. A heuristic for graph drawing. Congressus Numeratium, 42:149-160, 1984.

2. D.T. Lee, C.D. Yang, and C.K. Wong. Rectilinear paths among rectilinear obstacles. Discrete Appl. Math., 70:185-215, 1996.

3. G. Sander. A fast heuristic for hierarchical manhattan layout. In Proc. Symposium on Graph Drawing, LNCS 102\%, pages 447-458, 1996.

4. A. Vasiliu. A topological graph unfolding algorithm. In Proc. COMES'93, Douai, France, pages 73-76, 1993.

5. J.Q. Walker II. A node positioning algorithm for generalized trees. Software Pract. and Exper., 20(7):685-705, 1990. 